\title{
Évolution de la flore lactique thermophile au cours du pressage des fromages à pâte cuite
}

\author{
par \\ J. P. ACCOLAS, Monique VEAUX \\ Laboratoire de Technologie Laitière, I.N.R.A. \\ 78350 Jouy-en-Josas \\ et \\ L. VASSAL, G. MOCQUOT \\ Laiterie Expérimentale, I.N.R.A. \\ 78350 Jouy-en-Josas
}

\section{INTRODUCTION}

Les bactéries lactiques thermophiles, Streptococcus thermophilus et lactobacilles, jouent un rôle déterminant au cours du séjour du fromage sous presse, dans la fabrication du Gruyère et de l'Emmental. En effet, à ce stade, le développement du levain lactique thermophile qui va de pair avec son activité acidifiante favorise la synérèse du caillé, c'est-à-dire l'expulsion, au cours du pressage, d'une quantité appropriée de lactosérum. Aussi ce levain contribue-t-il de façon prépondérante à la réussite du fromage c'est-àdire à l'obtention, à la sortie de presse, le lendemain de la fabrication, d'un fromage " correctement » égoutté (extrait sec de 61 à 62 p. 100) et acidifié ( $\mathrm{pH}$ voisin de 5,2), ne contenant pas de lactose résiduel [11], toutes données qui ont été reconnues, par l'expérience, comme étant en corrélation étroite avec la bonne qualité du fromage.

Cela dit, peut-on considérer le jeune fromage comme une masse homogène dont l'évolution ultérieure serait sous la seule et étroite dépendance des conditions d'affinage : salage, température, humidité, composition de l'atmosphère ? Rien n'est moins sûr. Prenons l'exemple de "l'ouverture » qui revêt, sur le plan économique, une grande importance : le nombre, la dimension et la répartition des yeux à l'intérieur de la meule influencent notablement la valeur marchande du produit. Or, on constate que d'assez nombreux fromages présentent, après affinage, une ouverture plus abondante en périphérie qu'au centre alors que divers facteurs (teneur en sel plus élevée, teneur en humidité plus faible [8]) devaient normalement conduire à la situation inverse. Cette situation inverse est d'ailleurs 
aussi observée sur un pourcentage appréciable de meules de fromage. Tout ceci semble suggérer qu'une certaine hétérogénéité existe à l'intérieur du jeune fromage et qu'elle peut contribuer à orienter les fermentations ultérieures dans un sens ou dans un autre.

En particulier, il est maintenant connu que la teneur en lactate varie notablement d'un point à l'autre du jeune fromage et qu'elle peut, de ce fait, jouer un rôle dans la formation des yeux $[15,16]$. Ces variations de la teneur en lactate d'une zone à une autre du fromage peuvent trouver leur origine dans un développement plus ou moins abondant de la flore lactique thermophile suivant la zone considérée, comme le suggérait déjà en 1935 le travail de Burkey et al. [3]. C'est cette hypothèse que nous nous sommes attachés à vérifier, en faisant appel à des méthodes de dénombrement plus révélatrices que le comptage microscopique direct des cellules utilisé à l'époque par les auteurs américains. En outre, l'emploi de suspensions concentrées et congelées de bactéries lactiques au lieu des levains traditionnels [1], nous a permis de réaliser un ensemencement régulier du lait, d'une fabrication à une autre, ce qui constitue un avantage précieux sur le plan expérimental.

\section{METHODES}

L'étude a porté sur trente-sept meules de Gruyère fabriquées à la Laiterie Expérimentale de Jouy-en-Josas.

\section{Levains}

Le lait était ensemencé avec les levains suivants :

- un levain lactique mésophile constitué par un mélange de quatre souches appartenant aux espèces Streptococcus lactis, S. diacetylactis et $S$. cremoris. Ce levain était préparé sur lait écrémé autoclavé et ajouté au lait de fabrication à la dose de 0,06 p. 100 au moment du remplissage de la cuve de fabrication, c'est-à-dire environ 30 à $45 \mathrm{mn}$ avant l'emprésurage ;

- un levain lactique thermophile constitué par deux suspensions concentrées congelées, l'une de S. thermophilus CNRZ 302 et l'autre de Lactobacillus helveticus CNRZ 303, suspensions préparées selon des techniques décrites ailleurs [18]. Les doses d'inoculation étaient de $5 \mathrm{~g}$ de suspension de $S$. thermophilus et de $25 \mathrm{~g}$ de suspension de $L$. helveticus, pour 500 à 5501 de lait cru, c'est-à-dire le contenu d'une cuve de fabrication correspondant a un fromage. Au cours des essais, deux suspensions différentes de S. thermophilus et quatre de $L$. helveticus ont été successivement utilisées ;

- un levain de bactéries propioniques (Propionibacterium shermanii CNRZ 88), préparé sur lait écrémé additionné de $1 \mathrm{p} .100$ 
d'extrait de levure, était ajouté au lait de fabrication à la dose de 0,01 p. 100 .

\section{Fabrication des fromages}

Les meules de Gruyère étaient fabriquées selon la technique habituelle. Après ajustement du taux de matière grasse à environ $32 \mathrm{~g} / \mathrm{l}$, le lait était réchauffé à $32^{\circ} \mathrm{C}$ et ensemencé avec les différents levains (levains lactiques mésophile et thermophile et levain propionique). Le lait ensemencé était ensuite additionné de présure commerciale liquide (force 10000 ) de façon à obtenir une prise en $30 \mathrm{mn}$ environ. Le caillé était alors découpé, brassé et chauffé jusqu'à $54^{\circ} \mathrm{C}$ en $45 \mathrm{mn}$ environ. Le brassage était poursuivi pendant $40 \mathrm{mn}$; la température était abaissée à $49^{\circ} \mathrm{C}$ après $20 \mathrm{mn}$, par circulation d'eau froide dans la double paroi de la cuve. Après la mise en moule, les fromages étaient pressés pendant $21 \mathrm{~h}$ dans une pièce à $24^{\circ} \mathrm{C}$. On effectuait trois retournements, après $1 \mathrm{~h}, 3 \mathrm{~h}$ et $6 \mathrm{~h}$ de pressage. A la sortie de presse les meules de fromage (environ $60 \mathrm{~cm}$ de diamètre, $13 \mathrm{~cm}$ d'épaisseur, d'un poids de $44 \mathrm{~kg}$ ) étaient mises en cave froide $\left(12^{\circ} \mathrm{C}\right)$ où elles séjournaient 3 semaines environ avant le passage en cave chaude $\left(18^{\circ} \mathrm{C}\right)$.

\section{Echantillonnage}

Des prélèvements ont été faits sur le lait avant et après ensemencement de celui-ci avec les différents levains, sur le caillé mis sous presse, sur le fromage pendant le pressage, à la sortie de presse, $30 \mathrm{~h}$ et $48 \mathrm{~h}$ après la mise sous presse.

Les échantillons de fromage ont été prélevés (fig. 1) en périphérie de la meule au moyen d'une sonde stérile $n^{\circ} 5$ enfoncée radialement

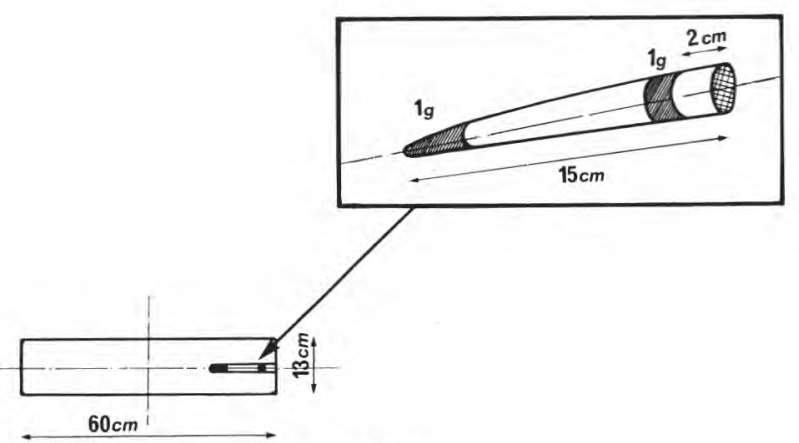

fig. 1

Prélèvement des échantillons dans la meule de fromage, pendant le pressage. 
dans le "talon " de la meule, à égale distance des deux faces du fromage, de façon à obtenir une " carotte " d'environ $15 \mathrm{~cm}$ de longueur. Nous avons effectué des dénombrements :

1. Sur l'échantillon ( $1 \mathrm{~g})$ représentatif de la zone périphérique du fromage, c'est-à-dire celui prélevé à $2 \mathrm{~cm}$ du bord extérieur de la meule.

2. Sur $1 \mathrm{~g}$ de fromage prélevé à l'extrémité interne de la sonde, représentatif de la zone centrale, située entre la moitié et le tiers du rayon du fromage.

A 1 g de fromage préalablement râpé stérilement, on ajoutait $0,2 \mathrm{~g}$ de citrate de sodium trisodique et une première fraction de $5 \mathrm{ml}$ d'eau distillée stérile. On homogénéisait à l'aide d'un broyeur Ultra-Turrax pendant $5 \mathrm{~s}$, puis on ajoutait une deuxième fraction de $5 \mathrm{ml}$ d'eau et on homogénéisait à nouveau. On répétait cette série d'opérations quatre fois en évitant tout échauffement excessif du liquide. On complétait à $100 \mathrm{ml}$ avec de l'eau et on obtenait ainsi une suspension de fromage diluée au 1/100 présentant une homogénéité satisfaisante. Le traitement avait aussi pour but de dissocier les amas bactériens et de fragmenter les formations bactériennes en chaîne, ce qui permettait de limiter les variations aléatoires du dénombrement, imputables à ces deux facteurs [9].

\section{Milieux de dénombrement}

Pour dénombrer $L$. helveticus, nous avons utilisé le milieu MRS ( Lactobacilli MRS broth » Difco n ${ }^{\circ} 881$ ), acidifié à l'aide d'acide acétique de façon que le $\mathrm{pH}$ après autoclavage soit de $5,35 \pm 0,05$, et gélosé à raison de 1,2 p. 100. Les ensemencements étaient effectués dans des boîtes de Petri. Après solidification de la première couche de gélose $(12 \mathrm{ml})$ mélangée à l'inoculum, on coulait une deuxième couche $(6 \mathrm{ml})$ de ce même milieu de façon à favoriser le développement des colonies de lactobacilles. Celles-ci sont dénombrables après $3 \mathrm{j}$ d'incubation à $42^{\circ} \mathrm{C}$. La souche de $S$. thermophilus étudiée ne forme pas de colonies visibles sur ce milieu aux dilutions habituellement utilisées.

Pour dénombrer $S$. thermophilus, nous avons utilisé le milieu ST qui contenait $(\mathrm{g} / \mathrm{l})$ : tryptone (Difco), 5 ; peptone (Evans), 5 ; $\mathrm{PO}_{4} \mathrm{HK}_{2}, 4$; saccharose, 10 ; gélatine, 2 ; acide citrique, 0,5 ; cystéine$\mathrm{HCl}, 0,2$; gélose, 12. Le $\mathrm{pH}$ était ajusté à 6,8 avant autoclavage. Les boîtes de Petri étaient incubées à $42^{\circ} \mathrm{C}$ pour éviter le développement éventuel des streptocoques lactiques mésophiles.

Sur ce milieu S. thermophilus 302 forme des petites colonies lenticulaires $\mathrm{S}$ de 1 à $2 \mathrm{~mm}$ de diamètre, dénombrables après $24 \mathrm{~h}$ d'incubation à $42^{\circ} \mathrm{C}$ et $L$. helveticus 303 , des petites colonies $\mathrm{R}$ (diamètre $\leqslant 0,5 \mathrm{~mm}$ ) d'aspect cotonneux, que l'on différencie aisément de celles de $S$. thermophilus. 


\section{Mesure des températures pendant le pressage}

On a utilisé deux sondes thermométriques constituées par des tubes métalliques creux de 1,8 $\mathrm{mm}$ de diamètre, renfermant chacun, à leur extrémité distale fermée, un thermocouple. Chaque sonde était enfoncée horizontalement dans le "talon » de la meule, à mi-distance des deux faces, au travers d'un trou percé dans le cercle en bois ceinturant la meule pendant le pressage. Les deux sondes étaient enfoncées de façon que les thermocouples soient situés l'un à $15 \mathrm{~cm}$ (centre) et l'autre à $2 \mathrm{~cm}$ (périphérie) du bord extérieur de la meule. Les deux thermocouples étaient reliés à un pyromètre enregistreur Speedomax type C (MECI, Paris).

\section{RESULTATS}

L'addition du levain lactique thermophile sous forme de suspensions concentrées de $S$. thermophilus et de $L$. helveticus conduit à un ensemencement du lait notablement supérieur à la flore naturelle du lait cru capable de former des colonies sur les deux milieux gélosés utilisés (tab. 1 et 2). Soulignons ici que les colonies formées par la flore dominante sur chacun de ces deux milieux étaient bien, nous l'avons vérifié, des colonies de $S$. thermophilus sur milieu ST et des colonies de L. helveticus sur milieu MRS acidifié. Le coefficient de variation observé* est de l'ordre de 37 p. 100 (milieu ST) et de 69 p. 100 (milieu MRS) dans le cas du lait additionné des levains thermophiles. Ce coefficient incluant l'erreur imputable à la méthode de dénombrement, on en déduit que l'emploi de suspensions concentrées permet d'obtenir, d'une fabrication à l'autre, une assez bonne régularité de l'ensemencement du lait.

Au moment de la mise sous presse, les dénombrements effectués sur le caillé montrent que celui-ci contient environ douze fois plus de $S$. thermophilus et environ sept fois plus de $L$. helveticus que le lait additionné du levain lactique. Quant au lactosérum, il contient à ce stade un nombre nettement plus faible de bactéries lactiques thermophiles : 105,380 S. thermophilus et 104,924 L. helveticus/g, moyennes établies sur cinq fabrications. Autrement dit, les bactéries du levain se retrouvent presque intégralement dans le caillé qui ne représente, à ce stade, que 10 p. 100 environ de la masse du lait mis en œuvre. On notera que le facteur de concentration de $S$. thermophilus, 11,9 , est du même ordre de grandeur que le rapport du poids

\footnotetext{
* L'écart-type calculé après transformation logarithmique à base 10 des données est en effet approximativement égal à 0,43 fois le coefficient de variation de la variable initiale [5].
} 


\section{TABLEAU 1}

Evolution de la flore lactique dénombrée sur milieu ST (S. thermophilus), au cours de la fabrication du Gruyère

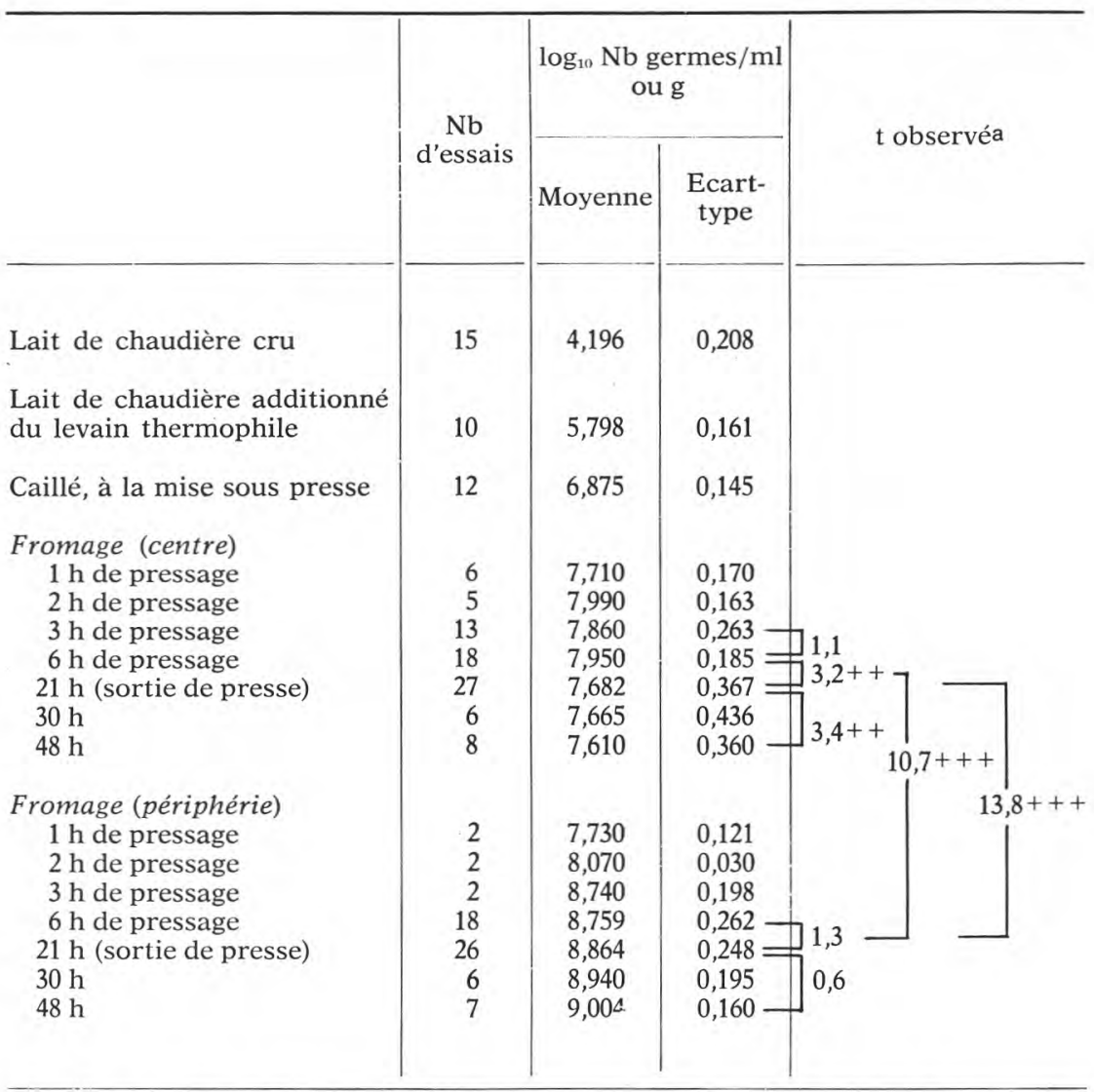

a : Epreuve t de Student. Les comparaisons portent soit sur les moyennes obtenues au centre ou en périphérie, après un temps identique de pressage, soit sur les moyennes obtenues dans la même zone après des durées différentes de pressage.

+++ : Différence hautement significative ( $t$ observé $>t 0,001$ ).

++ : Différence significative (t observé $>\mathrm{t} 0,01$ ).

+: Différence faiblement significative ( $t$ observé $>\mathrm{t} 0,05$ ).

du caillé à celui du lait utilisé, ce qui signifie que les streptocoques ne se sont pas beaucoup multipliés au cours du travail en chaudière. Dans le cas de L. helveticus, le facteur de concentration n'est que de 7,2 , ce qui traduit peut-être une moindre résistance des lactobacilles au chauffage du caillé en chaudière et explique vraisemblablement 


\section{TABLEAU 2}

Evolution de la flore lactique dénombrée sur milieu MRS acidifié (L. helveticus), au cours de la fabrication du Gruyère

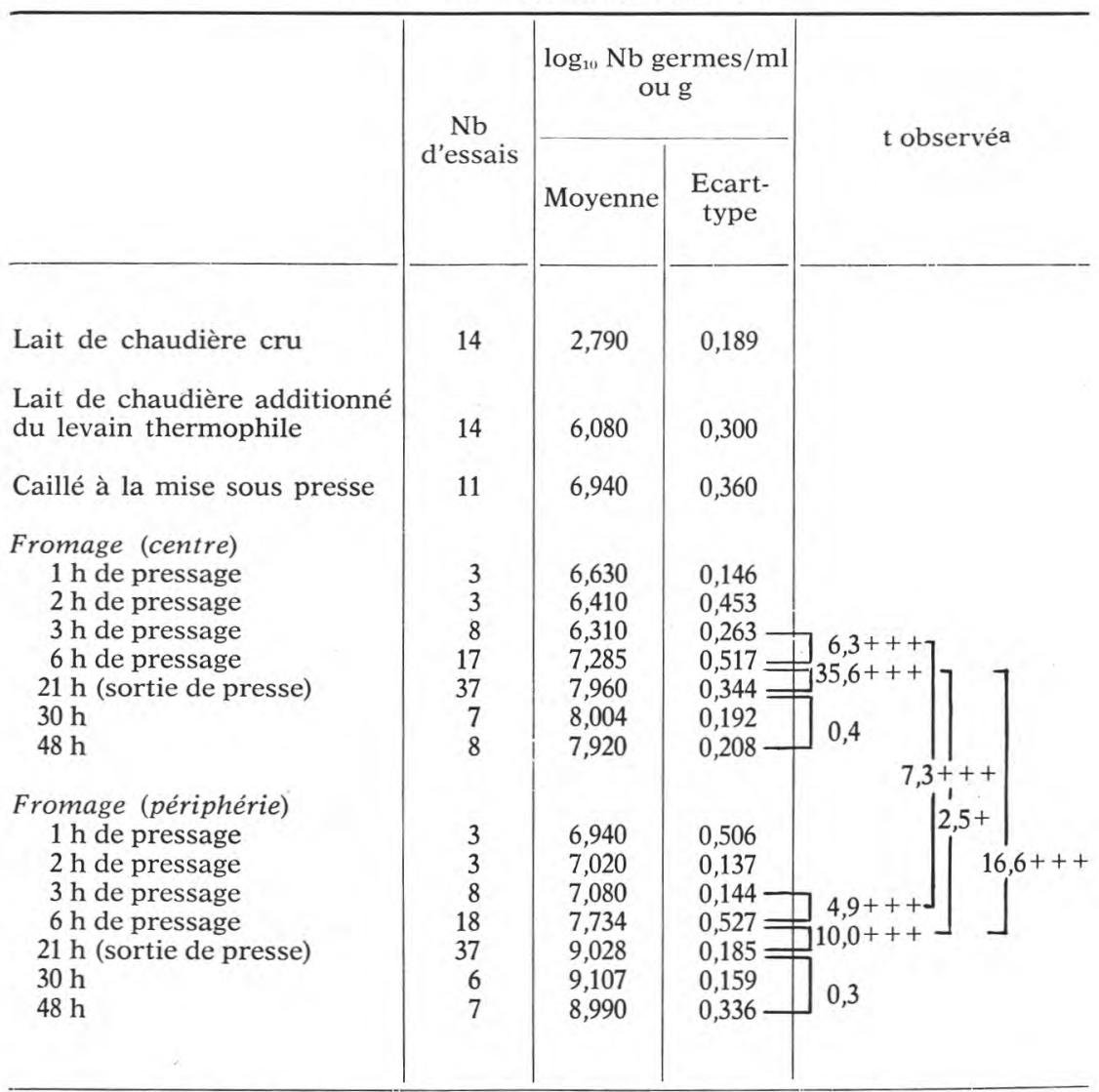

a: Epreuve $\mathrm{t}$ de Student. Voir légende tableau 1.

le décalage important qui se manifeste sous presse, entre le développement de $S$. thermophilus et celui de $L$. helveticus.

Comme le montrent les résultats présentés dans le tableau 1 et sur la figure 2, S. thermophilus, une fois le caillé mis sous presse, se développe rapidement. En effet, dès la $3^{\mathrm{e}} \mathrm{h}$ de pressage, il atteint une population maximale au centre du fromage et il est proche de celle qu'il atteindra en périphérie. Ensuite, le nombre de streptocoques ne varie pas notablement en périphérie, entre la $6^{\mathrm{e}} \mathrm{h}$ et la $21^{\mathrm{e}} \mathrm{h}$ 


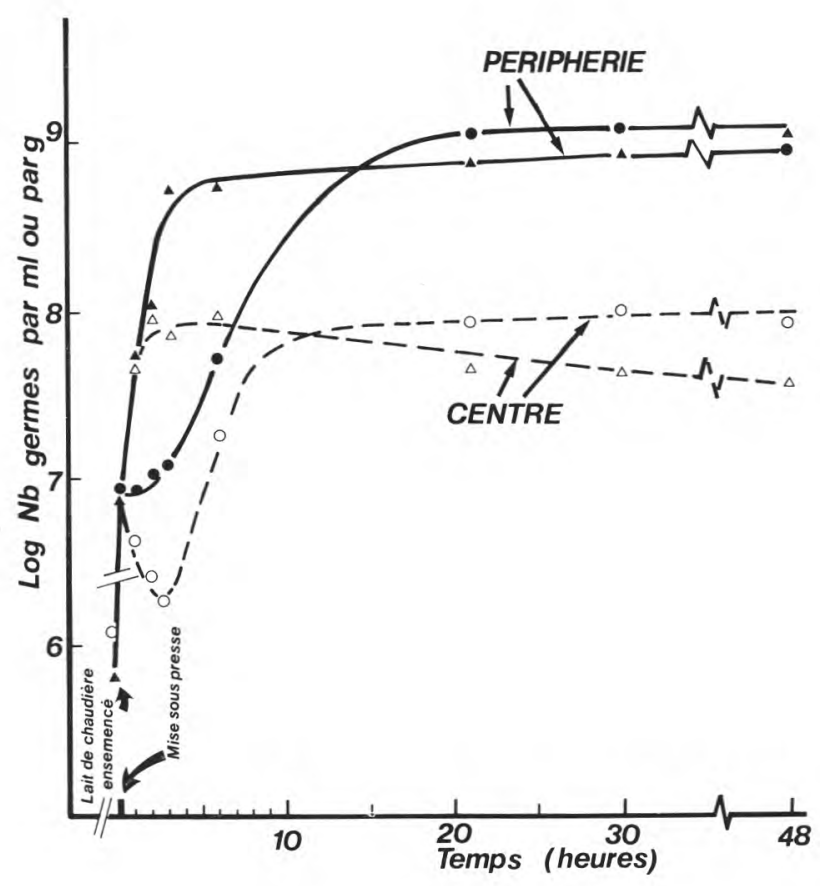

fig. 2

Evolution de la flore lactique thermophile au cours des $48 \mathrm{~h}$ suivant la mise sous presse du fromage.

Zone périphérique :

-— milieu MRS acidifié (L. helveticus).

$\Delta$ A milieu ST (S. thermophilus).

Zone centrale :

O....- 0 milieu MRS acidifié (L. helveticus).

$\triangle \ldots . . \triangle$ milieu ST (S. thermophilus).

de pressage, où il passe de 108,759 à 108,864 soit 1,27 fois plus, cette augmentation n'étant pas significative. Cette stabilité se confirme d'ailleurs ultérieurement dans les fromages âgés de $30 \mathrm{~h}$ et de $48 \mathrm{~h}$. Par contre, au centre du fromage, on observe une chute du nombre de streptocoques qui passe de 107,950 à 107,682 (1,85 fois moins) entre la $6^{\mathrm{e}} \mathrm{h}$ et la $21^{\mathrm{e}} \mathrm{h}$ de pressage ; cette diminution de près de 50 p. 100 est significative et se poursuit ultérieurement.

Dans le cas de L. helveticus (tab. 2 et fig. 2) on observe, au cours des 3 premières heures de pressage, une phase de latence peu marquée en périphérie mais une diminution notable du nombre de germes au centre du fromage. Les lactobacilles atteignent leur 
maximum beaucoup plus tard que $S$. thermophilus. Ainsi, entre la $6^{\mathrm{e}} \mathrm{h}$ et la $21^{\mathrm{e}} \mathrm{h}$ de pressage, leur nombre passe de 107,285 à 107,960 au centre (4,7 fois plus) et de 107,734 à 109,028 en périphérie (20 fois plus). Ces nombres restent à peu près stables dans chaque zone, chez les fromages âgés de $30 \mathrm{~h}$ et de $48 \mathrm{~h}$.

Il apparaît donc que la multiplication des deux germes est plus importante et, dans le cas de $S$. thermophilus, le nombre de germes plus stable en périphérie qu'au centre du fromage. Ainsi, après $20 \mathrm{~h}$ de pressage, les nombres de streptocoques et de lactobacilles sont respectivement 15,1 et 11,7 fois plus grands dans la zone périphérique que dans la zone centrale et les différences observées à ce stade sont hautement significatives (tab. 1 et 2 ).

Le fromage, qui est mis sous presse à une température de $49^{\circ} \mathrm{C}$, se refroidit plus vite en périphérie qu'au centre, au cours du pressage. Comme le montre la figure 3, la différence de température entre les deux zones dépasse $11^{\circ} \mathrm{C}$ après $6 \mathrm{~h}$ de pressage et est encore supérieure à $8^{\circ} \mathrm{C}$, lorsque le fromage est sorti de presse. Il en résulte que, suivant la zone du fromage et la période considérée du pressage, les conditions de température sont plus ou moins favo-

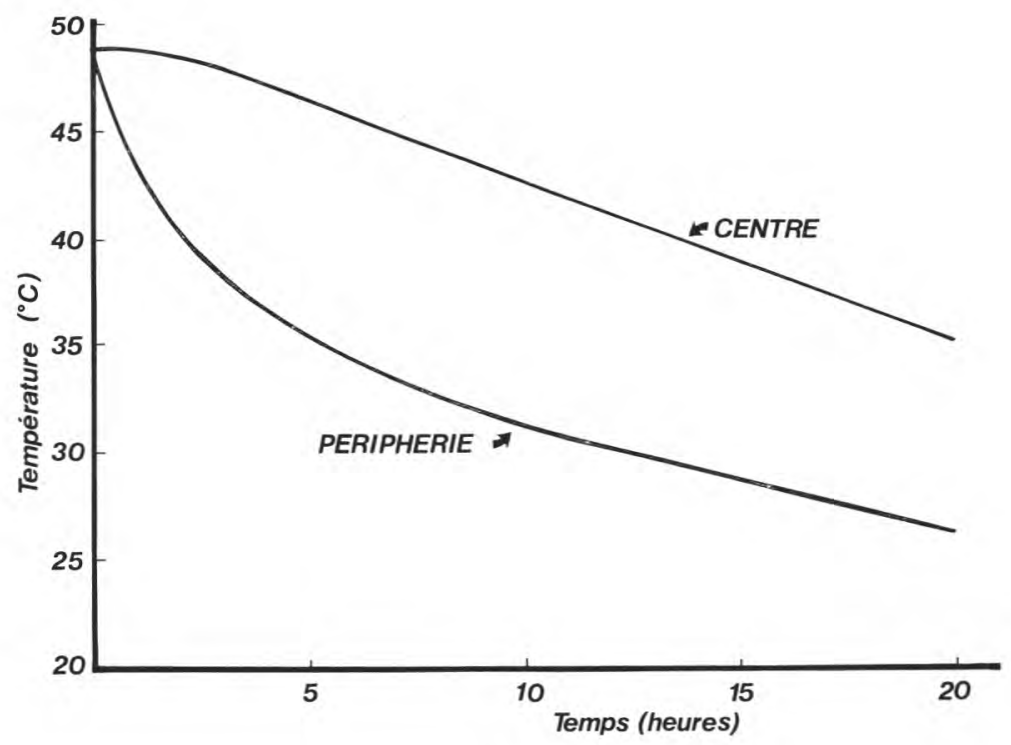

fig. 3

Evolution des températures, au centre et à la périphérie du fromage, pendant le pressage.

Température ambiante: $24^{\circ} \mathrm{C}$. 
rables à la fermentation lactique. En effet, on a mesuré (dans du lait préalablement chauffé à $80^{\circ} \mathrm{C}$ pendant $30 \mathrm{mn}$ ) l'activité acidifiante du levain de $S$. thermophilus et de $L$. helveticus en fonction de la température d'incubation. Les résultats figurent au tableau 3 et montrent que la quantité d'acide formée dans ces conditions est maximale à $44^{\circ} \mathrm{C}$ et diminue à des températures plus basses ou plus élevées.

\section{TABLEAU 3}

Quantité d'acide produite dans du lait par une culture mixte de S. thermophilus 302 et de L. helveticus 303, en fonction de la température d'incubation.

Milieu de culture : lait préalablement chauffé à $80^{\circ} \mathrm{C}$ pendant $30 \mathrm{mn}$. Inoculum : 0,5 p. 100 de chaque souche. Durée de l'incubation : $6 \mathrm{~h}$.

\begin{tabular}{c|c|c|c|c|c|c|c|c}
\hline $\begin{array}{c}\text { Température d'incubation } \\
\left({ }^{\circ} \mathrm{C}\right)\end{array}$ & 35 & 38 & 40 & 42 & 44 & 46 & 48 & 50 \\
\hline & & & & & & & & \\
\hline & & & & & & & \\
Quantité d'acide formée (\%) & 0,45 & 0,55 & 0,70 & 0,80 & 0,88 & 0,85 & 0,66 & 0,26 \\
\hline
\end{tabular}

\section{DISCUSSION}

Les résultats obtenus appellent un certain nombre de remarques et de commentaires. Le décalage dans le temps que nous avons observé dans le développement des deux espèces bactériennes du levain thermophile, confirme les données établies par Steffen et Blanc [13]. Ces auteurs, en étudiant l'évolution sous presse de la teneur en acide lactique de fromages expérimentaux, ont en effet montré qu'avec un levain contenant uniquement $S$. thermophilus, l'acidification était rapide au cours des premières heures de pressage mais plafonnait dès la $8^{\mathrm{e}} \mathrm{h}$ à une valeur anormalement basse. Inversement, lorsqu'ils utilisaient un levain ne contenant que des lactobacilles (soit L. helveticus, soit $L$. lactis), l'acidification démarrait lentement mais se poursuivait tout au long du pressage. Ainsi, avec $L$. helveticus, la teneur en acide lactique était environ 4,5 fois plus élevée à la sortie de presse qu'à la $8^{\mathrm{e}} \mathrm{h}$ de pressage et, avec L. lactis, presque 2 fois plus élevée. Dans ces conditions, il n'est pas sûr que la synergie, qui est à l'origine d'une production d'acide très active lorsque $S$. thermophilus et $L$. helveticus sont cultivés sur lait dans des conditions favorables au développement des deux germes [2], joue de façon comparable dans le fromage. Cette remarque vaut en particulier pour la zone centrale où le développement des deux bactéries lactiques est à la fois très décalé dans le temps et limité. 
D'autre part, on peut penser que la quantité d'enzymes libérées par les bactéries lactiques du levain et susceptibles de jouer un rôle au cours de l'affinage [17], notamment en ce qui concerne l'activité de la fermentation propionique, varie notablement d'une zone à l'autre du fromage.

Enfin, les différences de développement observées entre centre et périphérie montrent qu'il existe, selon les zones considérées dans le fromage, des conditions plus ou moins favorables aux bactéries lactiques et il convient maintenant d'analyser les facteurs limitants en jeu.

Il est bien connu que l'un des facteurs qui gouvernent le développement des bactéries lactiques thermophiles au cours du pressage du fromage est la température. Rappelons que Burkey et al. [3] ont été les premiers à montrer qu'il se crée, sous presse, un gradient de température dans la masse du fromage. La périphérie se refroidit plus vite que l'intérieur et en conséquence la fermentation lactique démarre plus rapidement en périphérie qu'à l'intérieur du fromage. Cela a été confirmé par Steffen et al. [14]. Dans notre cas, on a observé que l'ajustement de la température du mélange de caillé et de lactosérum à $49^{\circ} \mathrm{C}$, avant la mise en moule, conduit à l'établissement du gradient de température représenté sur la figure 3. L'existence d'un gradient de température plus favorable à la fermentation lactique dans une zone du fromage que dans l'autre [16] rend compte, sans doute pour une bonne part, des différences de développement des bactéries lactiques ainsi que le confirme le tableau 3 et on a d'ailleurs montré que la teneur en acide lactique est plus élevée en périphérie qu'au centre du fromage $[15,16]$.

Ces variations de la teneur en acide lactique peuvent s'expliquer de la manière suivante : vers la périphérie du fromage où débute la fermentation lactique, on assiste vraisemblablement au phénomène décrit par Dolby [6], puis par Czulak et al. [4]. Ces auteurs ont observé que, dans le caillé de Cheddar qui renferme la grande majorité des bactéries lactiques, la fermentation du lactose en acide lactique est plus active que dans le lactosérum, bien plus pauvre en bactéries lactiques. Dans ces conditions, la teneur en lactose diminue plus vite dans l'eau du caillé que dans le lactosérum et ceci a pour conséquence une diffusion du lactose du lactosérum vers le caillé, favorisant une poursuite de la fermentation lactique dans ce dernier.

Nous pensons que le même phénomène peut être transposé, pour le Gruyère, non plus au lactosérum et au caillé mais à la meule entière sous presse : à la périphérie de cette meule, en raison de la température plus favorable à la fermentation qui y règne au moins pendant les premières heures de pressage, les bactéries lactiques sont plus nombreuses et plus actives qu'au centre du fromage (fig. 2). Il s'ensuit que la teneur en lactose de la périphérie doit diminuer plus vite que celle du centre, provoquant ainsi une diffusion du lactose 
du centre vers la périphérie. Dans ces conditions, la teneur en lactose diminue rapidement dans la zone centrale, pendant les premières heures de pressage et la fermentation lactique se poursuit activement en périphérie grâce à l'afflux de lactose. On sait que l'acide lactique produit est évacué en partie avec le lactosérum d'égouttage. Est-il possible qu'une autre partie de l'acide lactique formé en périphérie diffuse vers le centre du fromage, là où il est produit plus lentement ? On peut le supposer car cela empêcherait l'établissement d'un gradient de concentration en acide lactique trop important dans la masse du fromage. En outre, l'appauvrissement rapide de la teneur en lactose et la chute du $\mathrm{pH}$ (résultant de la diffusion de l'acide lactique) au centre du fromage permettraient conjointement d'expliquer pourquoi les bactéries lactiques du levain voient leur croissance entravée dans cette partie du fromage, même au moment où la température y devient favorable à leur développement (fig. 2).

Toutefois, Czulak et al. [4], dans le cas du Cheddar, ont également observé que la diffusion de l'acide lactique, produit dans le caillé, vers le lactosérum, est relativement lente. Il se pourrait également qu'une fraction de cet acide soit fixée par les protéines du fromage [12] dans la zone même où cet acide est produit, surtout au début de la fermentation quand le $\mathrm{pH}$ est encore élevé (au-dessus de 5,7 par exemple). L'une et l'autre causes (lenteur de la diffusion, fixation partielle) expliqueraient l'accumulation de lactate observée vers la périphérie de la meule $[15,16]$.

Si l'on tient compte de cette possibilité de fixation de l'acide lactique par les protéines, les teneurs plus fortes en lactate observées à la périphérie du fromage peuvent également résulter, pour une part, du fait que l'acide lactique est formé en périphérie dès le début du pressage, dans un caillé possédant une teneur en eau encore assez forte (proche de 50 p. 100 [7]), donc relativement riche en lactose et capable d'accumuler la quantité correspondante d'acide lactique. Par contre entre 10 et $20 \mathrm{~h}$ de pressage, au moment où la fermentation pourrait, grâce à la température favorable, être active en zone centrale, le caillé renferme environ 40 p. 100 d'eau, ce qui correspond à une teneur en lactose, donc finalement en acide lactique par unité de poids, nettement plus faible [10].

En tout état de cause, on peut considérer le fromage sous presse comme un " écosystème » où interviennent, en fonction du temps, le gradient de température du centre vers la périphérie du fromage, le nombre et l'activité des streptocoques et des lactobacilles thermophiles et l'effet stimulant que chacune de ces espèces exerce ou non, selon les circonstances, vis-à-vis de l'autre.

Il est loisible de penser qu'un tel écosystème peut engendrer toute une diversité " d'équilibres » (acidification lente ou rapide) et que les modalités du procédé technologique (température du caillé 
à la sortie de la cuve, vitesse de refroidissement en fonction de la température ambiante et des dimensions de la meule, vitesse d'égouttage, etc.) exercent, dans ce domaine, une influence notable, comme Czulak et al. [4] l'ont déjà montré dans le cas du Cheddar. En outre, un équilibre une fois obtenu, caractérisé par un certain gradient de lactate dans les diverses parties de la meule, il reste à déterminer comment évolue ce gradient au cours de l'affinage et à apprécier son influence sur le déroulement de la fermentation propionique et la formation des yeux.

Cela dit, la diversité d'équilibres évoquée ci-dessus doit cependant être limitée dans le cas d'une fabrication "normale » de Gruyère, c'est-à-dire une fabrication susceptible de conduire plus tard à un fromage affiné de bonne qualité. En effet, l'expérience a montré que dans un tel fromage à la sortie de presse : a) le sucre est intégralement transformé en acide lactique, y compris dans la partie centrale de la meule [17] et b) la teneur moyenne en acide lactique par gramme de fromage correspond à peu de chose près à la teneur en lactose que posséderait un caillé de même teneur en eau que le fromage sortant de presse (38 à $40 \mathrm{p} .100)$, en l'absence de toute fermentation lactique $[10,17]$.

\section{Remerciements}

Nous remercions très vivement $M$. Bouillanne d'avoir préparé les suspensions concentrées de bactéries lactiques thermophiles utilisées au cours de ce travail et $M$. Pitel qui a fabriqué les Gruyères. Nous sommes reconnaissants à M. Auclair des critiques et des suggestions qu'il nous a formulées au cours de la rédaction $d u$ manuscrit.

\section{Rés u m é}

Au cours du séjour sous presse des fromages à pâte cuite, $S$. thermophilus se développe assez rapidement et atteint une population maximale avant la $6^{\mathrm{e}} \mathrm{h}$ de pressage alors que $L$. helveticus se développe beaucoup plus lentement et n'atteint son maximum que tardivement, entre la $15^{\mathrm{e}} \mathrm{h}$ et la $20^{\mathrm{e}} \mathrm{h}$ de pressage.

Des différences notables, supérieures à un log décimal, ont été mises en évidence entre les populations bactériennes formées au centre et à la périphérie d'un même fromage.

Les conséquences de ce phénomène sur l'établissement d'un gradient de concentration en lactate dans la masse du fromage, au cours de l'égouttage, sont discutées. 


\section{S u m m a r y}

\section{DEVELOPMENT OF THE THERMOPHILIC LACTIC ACID BACTERIA OF HARD CHEESES DURING PRESSING}

During whey drainage under press of hard cheeses (Gruyère \& Emmental), S. thermophilus grows fairly rapidly and reaches its maximum in less than 6 hours of pressing, whereas L. helveticus grows much more slowly and reaches its maximum later, betwen 15 and 20 hours of pressing.

Noticeable differences have been observed (higher than a decimal $\log$ ), between the bacterial populations developed in the center and at the edge of the same cheese.

The implications of this phenomenon in the formation of a lactate gradient within the cheese, during whey drainage, are discussed.

\section{Bibliographie}

[1] Accolas (J. P.), Auclair (J.), Bouillanne (C.), Mocouot (G.), Rousseaux (P.), Valles (E.), VASSAL (L.) (1970). - Préparation et utilisation en fromagerie de suspensions concentrées et congelées de bactéries lactiques. XVIII ${ }^{\mathrm{e}}$ Congr. Int. Lait., $1 \mathrm{~F}, 281$.

[2] Accolas (J. P.), Veaux (M.), Auclair (J.) (1971), - Etude des interactions entre diverses bactéries lactiques thermophiles et mésophiles, en relation avec la fabrication des fromages à pâte cuite. Le Lait, 51, 249-272.

[3] Burkey (L. A.), Sanders (G. P.), Matheson (K. J.) (1935). - The bacteriology of Swiss cheese. IV. Effect of temperature upon bacterial activity and drainage in the press, J. Dairy Sci., 18, 719-731.

[4] Czulak (J.), Conochie (J.), Sutherland (B. J.), Van Leeuwen (H. J. M.) (1969). - Lactose, lactic acid and mineral equilibria in Cheddar cheese manufacture. J. Dairy Res., 36, 93-101.

[5] Dagnelie (P.) (1973). - Théorie et méthodes statistiques : applications agronomiques. I, p. 185-186, $2^{\circ}$ éd., Presses Agronomiques de Gembloux.

[6] Dolby (R. M.) (1941). - The control of acid development in Cheddar cheesemaking. N.Z.J. Sci. Technol., A22, 289-302.

[7] Kosıкоwsкi (F. V.), Mocouot (G.) (1958), - Progrès de la technologie du fromage. Etudes agricoles $\mathrm{n}^{\circ} 38$, F.A.O., Rome.

[8] Langsrud (T.), Reinbold (G. W.) (1973). - Flavor development and microbiology of Swiss cheese. A review. J. Milk Food Technol., 36, 531-542 et 593-609.

[9] Martley (F. G.) (1972). - The effect of cell numbers in streptococcal chains on plate-counting. N.Z.J. Dairy Sci. Technol., 7, 7-11.

[10] Mocouot (G.) (1947). - Etude sur l'eau liée des fromages. Le Lait, 27, 576-595.

[11] Mocouot (G.) (1971). - Occurrence and role of microorganisms in cheese. International Symposium on conversion and manufacture of foodstuffs by microorganisms, Kyoto, 191-197. 
[12] Ribadeau-Dumas (B.) (1977). - Communication personnelle.

[13] StEFFen (C.), Blanc (B.) (1971). - Die Milchsäure und deren Konfiguration in Emmentalerkäse. Schweiz. Milchztg., 97 (WB no 126), 1079-1084.

[14] StefFen (C.), Sollberger (H.), BURI (K.) (1972). - Rapports de température durant la fermentation lactique du Gruyère. Laitier Romand, 48 ( $\mathrm{n}^{\circ}$ 103-104), $1-8$.

[15] Steffen (C.), Nick (B.) (1974). - Evolution de la fermentation lactique et de la dégradation de l'acide lactique dans diverses zones de l'Emmental. $\mathrm{XIX}^{\mathrm{e}}$ Congr. Int. Lait., 1F, 329-330.

[16] StefFen (C.) (1975). - Enzymatische Bestimmungsmethoden zur Erfassung der Gärungsvorgänge in der milchwirschaftlichen Technologie. Lebensmitt.Wiss. und-Techn., 8, 1-16.

[17] StefFen (C.) (1977). - Kessiunabhängige Kulturen. Schweiz. Michztg., 193, 334-335, 364 und 380.

[18] Valles (E.), Mocouot (G.) (1968). - Préparation de suspensions concentrées et congelées de bactéries lactiques thermophiles destinées à la fromagerie. Le Lait, 48, 631-643. 